\title{
Choline Acetyltransferase, Glutamic Acid Decarboxylase and Somatostatin in the Kainic Acid Model for Chronic Temporal Lobe Epilepsy
}

\author{
Halina Baran ${ }^{a, b}$ Berthold Kepplinger ${ }^{c, d}$ Markus Draxler ${ }^{a, b}$ \\ Gerhard Skofitsch ${ }^{\mathrm{e}}$ \\ a Institute of Pharmacology and Toxicology, and ${ }^{b}$ Institute of Physiology, Veterinary University Vienna, Vienna, \\ 'Department of Neurology, Diagnostic and Therapy Centre, Ostarrichiklinikum, Neuropsychiatric Hospital \\ Mauer/Amstetten, Mauer/Amstetten, dDepartment of Neurology, Klinikum Mostviertel, General Hospital Amstetten, \\ Amstetten, and Institute of Zoology, Karl Franzens University Graz, Graz, Austria
}

\section{Key Words \\ Kainic acid - Chronic temporal lobe epilepsy model • Glutamic acid decarboxylase $\cdot$ Choline acetyltransferase • Somatostatin - Cortical regions - Neuroprotection • Neurodegeneration - Epileptogenesis · Brain · Rat}

\begin{abstract}
The aim of the study was to investigate neurochemical changes in a kainic acid (KA; $10 \mathrm{mg} / \mathrm{kg}$, s.c.)-induced spontaneous recurrent seizure model of epilepsy, 6 months after the initial KA-induced seizures. The neuronal markers of cholinergic and $\gamma$-aminobutyric acid (GABA)ergic systems, i.e. choline acetyltransferase (ChAT) and glutamic acid decarboxylase (GAD) activities, and a marker for neuropeptide, i.e. level of somatostatin, have been investigated. The brain regions investigated were the hippocampus, amygdala/piriform cortex, caudate nucleus, substantia nigra and the frontal, parietal, temporal and occipital cortices. Six months after KA injection, reduced ChAT activity was observed in the amygdala/piriform cortex (47\% of control; $p<0.001$ ), increased ChAT activity in the hippocampus $(119 \%$ of control; $p<0.01$ ) and normal ChAT activity in the other
\end{abstract}

brain regions. The activity of GAD was significantly increased in all analysed cortical regions (between 146 and $171 \%$ of control), in the caudate nucleus $(144 \%$ of control; $p<0.01)$ and in the substantia nigra $(126 \%$ of control; $p<0.01$ ), whereas in the amygdala/piriform cortex, the GAD activity was moderately lowered. The somatostatin level was significantly increased in all cortical regions (between 162 and $221 \%$ of control) as well as in the hippocampus (119\% of control), but reduced in the amygdala/piriform cortex ( $45 \%$ of control; $p<0.01$ ). Six months after KA injection, the somatostatin:GAD ratio was lowered in the amygdala/piriform cortex $(49 \%$ of control) and in the caudate nucleus ( $41 \%$ of control), whereas it was normal in the hippocampus and moderately increased in the cortical brain regions. A positive correlation was found between seizure severity and the reduction of both ChAT activities and somatostatin levels in the amygdala/piriform cortex. The results show a specific pattern of changes for cholinergic, GABAergic and somatostatinergic activities in the chronic KA model for epilepsy. The revealed data suggest a functional role for them in the new network that follows spontaneous repetitive seizures.

Copyright $\odot 2004$ S. Karger AG, Base

\begin{tabular}{ll}
\hline KARGER & ( 2004 S. Karger AG, Basel \\
Fax +4161306 1234 & 1424-862X/04/0136-0290\$21.00/0 \\
$\begin{array}{l}\text { E-Mail karger@karger.ch } \\
\text { www.karger.com }\end{array}$ & $\begin{array}{l}\text { Accessible online at: } \\
\text { www.karger.com/nsg }\end{array}$
\end{tabular}

Halina Baran, $\mathrm{PhD}$

Institute of Pharmacology and Toxicology

Veterinary University Vienna

AT-1210 Vienna (Austria)

Tel. +43 125077 4519, Fax +43 125077 4590, E-Mail Halina.Baran@vu-wien.ac.at 


\section{Introduction}

The behavioural alterations in the rats and the neurochemical and histopathological changes in their brains after systemic kainic acid (KA) application show, at least in part, similarities to the behavioural, neurochemical and histopathological alterations observed in rats with amygdala-kindled seizures and complex partial seizures or temporal lobe epilepsy in humans [1-7]. KA serves as the prototype pharmacological agonist for pre- and postsynaptic, non-N-methyl- $D$-aspartate KA subtypes of glutamate excitatory amino acid receptors [2, 3, 8]. KAinduced seizures cause lesions consisting in a degeneration of the nerve cells of limbic brain regions, tissue necrosis and haemorrhages involving the amygdala, piriform cortex, entorhinal cortex, hippocampus and other limbic structures [2-5, 9-13]. Previous neurochemical investigations revealed significant changes characterised by early enhancement of the prostaglandin synthesis [14], increased turnover of mono-aminergic neurotransmitters $[4,15]$ and a reduction of neuropeptide somatostatin [16] in the acute phase $(3 \mathrm{~h})$ after KA injection. Later, a selective reduction of glutamic acid decarboxylase (GAD) activity was observed in the hippocampus and the amygdala/piriform cortex as well as a reduction of choline acetyltransferase (ChAT) activity in the amygdala/piriform cortex complex 3 days after $\mathrm{KA}$ injection, indicating a destruction of cholinergic and $\gamma$-aminobutyric acid (GABA)ergic neurons in these brain regions [4]. One month after KA injection, a recovery of GAD activities in the hippocampus was observed [4], suggesting the possibility of regenerative processes in this area. A regeneration of GABAergic activity has been reported due to sprouting of GABAergic neurons after KA-induced neurodegeneration in the hippocampus [17] and furthermore, an increased GAD mRNA level in a rat model of temporal lobe epilepsy has been demonstrated [18]. Two months after KA injection, increased GAD activity in the frontal cortex was found [19]. In human studies, both increase and decrease in GABAergic activities in the epileptic tissues have been documented [20-23]. Therefore, it is very likely that the type and extent of axonal sprouting modify the brain excitability and also affect the biochemical molecular processes in the epileptic brain. Electrophysiological studies have shown recurrence of spontaneous seizures after subcutaneous, intraperitoneal or intracerebral KA injection [1, 24, 25]. The present study aims at the investigation of the involvement of ChAT and GAD activities in the brain of rats with spontaneous recurrent seizures 6 months after KA administration.
Since somatostatin is a cotransmitter of GABAergic neurons [26] and plays a role in modulation and/or propagation of seizures [27-29], we also analysed the somatostatin levels. Part of the study has been presented in abstract form [30].

\section{Materials and Methods}

\section{Animals and Materials}

Male Sprague-Dawley rats (Forschungsinstitut für Versuchstierzucht, Himberg, Austria) of 280-320 g body weight were used. The animals were housed in groups of 4-5 per cage, in a room with a controlled light/dark cycle ( $12 \mathrm{~h}$ light/12 h dark), and were given free access to laboratory chow and tap water. All chemicals were obtained from commercial suppliers and were of the highest purity available.

KA (Sigma, USA) dissolved in saline at $10 \mathrm{mg} / \mathrm{ml}$ and adjusted to $\mathrm{pH} 7.0$ was administered subcutaneously in the neck area at a dose of $10 \mathrm{mg} / \mathrm{kg}$. The control animals were treated with the corresponding amount of saline. The injections were given in the morning, between 9 and 10 a.m.

\section{Evaluation of the Behavioural Changes after KA Treatment}

According to the rating scale previously described by Sperk et al. [4], the behaviour of each animal was continuously evaluated during $3 \mathrm{~h}$ after KA or vehicle injection. The following scores were used for rating the severity of KA-induced seizures: $0=$ normal, rare wet dog shakes (WDS), no convulsions; 1 = intermediate number of WDS, staring, rare focal convulsions affecting head and extremities; $2=$ frequent WDS, frequent focal convulsions, eye closure, sniffing, facial clonus, staring; $3=$ frequent WDS, frequent convulsions, head nodding associated with more severe facial clonus, unilateral or bilateral forelimb clonus with rearing and salivation (but without falling); $4=$ continuous generalised seizures, rearing with loss of balance and falling accompanied by generalised clonic seizures; $5=$ sustained generalised clonic convulsions, frequently death within $3 \mathrm{~h}$.

For the 6-month experiment, KA-treated rats showing developed seizures, i.e. rating 3 and 4 , were used. The behaviour of the KAtreated animals $(n=15)$ and control animals $(n=10)$ was observed every Monday and Wednesday from 9 to 10 a.m. and their behaviour was evaluated by counting the occurrences of WDS, focal epileptic activities and spontaneous seizures. During the 6-month period, 4 KA-treated animals died due to status epilepticus.

\section{Dissection and Preparation of Homogenate}

Six months after KA $(10 \mathrm{mg} / \mathrm{kg}$, s.c. $)$ or saline administration, the rats were decapitated, the brains were quickly removed and the frontal cortex, cingulate cortex, occipital cortex, parietal cortex, temporal cortex, amygdala/piriform cortex, dorsal hippocampus, nucleus caudatus and substantia nigra were dissected according to the dissection guides [31, 32]. The dissected areas were immediately frozen, weighted and stored for up to 2 weeks at $-70^{\circ} \mathrm{C}$.

The brain areas were homogenised by sonicating in $20 \mathrm{vol}$ of $\mathrm{H}_{2} \mathrm{O} / \mathrm{N}_{2}\left(0^{\circ} \mathrm{C}\right)$ and the homogenates were divided into three aliquots. Two aliquots were used immediately for GAD and ChAT measurement; the third aliquot was prepared for somatostatin determination. 
Table 1. Occurrence of focal convulsions and spontaneous seizures in KA-treated rats during 6 months after KA administration $(10 \mathrm{mg}$ / $\mathrm{kg}$, s.c.)

\begin{tabular}{lll}
\hline $\begin{array}{l}\text { Time period after } \\
\text { KA injection }\end{array}$ & $\begin{array}{l}\text { Focal epileptic } \\
\text { activities per rat }\end{array}$ & $\begin{array}{l}\text { Spontaneous } \\
\text { seizures per rat }\end{array}$ \\
\hline 0-1 month & $1.3 \pm 0.34$ & $0.0 \pm 0.0$ \\
1-2 month & $1.2 \pm 0.39^{*}$ & $0.5 \pm 0.17^{*}$ \\
2-3 month & $0.5 \pm 0.17^{*}$ & $0.6 \pm 0.16^{*}$ \\
3-4 month & $0.0 \pm 0.0$ & $1.6 \pm 0.27^{*}$ \\
4-5 month & $0.0 \pm 0.0$ & $0.6 \pm 0.16^{*}$ \\
5-6 month & $0.0 \pm 0.0$ & $3.3 \pm 0.26^{*}$ \\
F & 9.529 & 160.672 \\
p & 0.006 & $<0.001$ \\
\hline
\end{tabular}

Focal convulsions and spontaneous seizures were calculated for each month after KA administration. The data represent means \pm SEM of $11 \mathrm{KA}$-treated animals.

$* \mathrm{p}<0.05$ (one-way ANOVA).

\section{Determination of GAD Activity}

GAD activity, as a marker for GABAergic neurons, was measured by trapping $\left[{ }^{14} \mathrm{C}\right] \mathrm{CO}_{2}$ formed from L- $1\left[{ }^{14} \mathrm{C}\right]$ glutamic acid (Amersham) as described by Roberts and Simonsen [33]. The homogenates were diluted with $\mathrm{H}_{2} \mathrm{O} / \mathrm{N}_{2}$ to a final concentration of 1:100 and centrifuged at $15,000 \mathrm{~g}$ for $10 \mathrm{~min}$. The incubation mixture contained $100 \mu$ of supernatant, $0.1 M$ imidazole- $\mathrm{HCl}$ buffer, $\mathrm{pH}$ 7.1, $0.5 \mathrm{~m} M$ pyridoxal phosphate (Sigma) and $4 \mathrm{~m} M\left[{ }^{14} \mathrm{C}\right]$ glutamic acid $\left(0.1 \mathrm{mCi} / \mathrm{mmol}\right.$; Amersham), saturated with $\mathrm{N}_{2}$ at a final volume of $200 \mu$ l. The incubation was carried out in polycarbonate tubes covered with a rubber stopper top (Kontes, New Jersey), exposing a small plastic basket in which a small piece of filter paper $(11 \times$ $90 \mathrm{~mm}$ ) soaked with $100 \mu \mathrm{l}$ hyamine hydroxide (Packard) was placed. The vials were incubated at $37^{\circ} \mathrm{C}$ for $1 \mathrm{~h}$. The reaction was stopped by putting the tubes on ice and injecting $0.5 \mathrm{ml}$ of $0.3 \mathrm{M}$ perchloric acid through the rubber cap. The vials were incubated again at $37^{\circ} \mathrm{C}$ for $1 \mathrm{~h}$ to allow $\left[{ }^{14} \mathrm{C}\right] \mathrm{CO}_{2}$ to develop and to be trapped on the filter paper. Finally, the filter papers were transferred to polyethylene vials containing $8 \mathrm{ml}$ scintillation fluid and processed for liquid scintillation counting.

\section{Determination of ChAT Activity}

ChAT activity, as a marker for cholinergic neurons, was determined by the rate of formation of $\left[{ }^{14} \mathrm{C}\right]$ acetylcholine from choline and ${ }^{14} \mathrm{C}$-labelled coenzyme $\mathrm{A}$ according to the procedure described by Fonnum [34]. The homogenate was diluted with $10 \mathrm{~m} M$ EDTA, $0.5 \%$ Triton X-100, $30 \mathrm{~m} M$ sodium phosphate buffer, $\mathrm{pH} 7.4$, to yield a final dilution of 1:150. An aliquot of the homogenate $(40 \mu \mathrm{l})$ was incubated with $30 \mathrm{~m} M \mathrm{NaCl}, 20 \mathrm{~m} M$ EGTA, $8 \mathrm{~m} M$ choline chloride, $0.2 \mathrm{mM}\left[{ }^{14} \mathrm{C}\right]$ acetyl coenzyme A $(0.8 \mathrm{mCi} / \mathrm{mmol}$; Amersham $)$ and sodium phosphate buffer, $\mathrm{pH} 7.4$, at $37^{\circ} \mathrm{C}$ for $30 \mathrm{~min}$. The final volume was $130 \mu$ l. The reaction was stopped by adding $5 \mathrm{ml}$ ice-cold $10 \mathrm{~m} M$ sodium phosphate buffer, $\mathrm{pH} 7.4$, and placing the tubes on ice. The labelled acetylcholine formed during the reaction was extracted with $1 \mathrm{ml}$ heptanon-3 containing $1 \%$ tetraphenylborate. After vigorous shaking, the tubes were centrifuged at $5,000 \mathrm{~g}$ for
$10 \mathrm{~min}$ and $500 \mu \mathrm{l}$ aliquots of the organic phase were transferred to counting vials and processed for liquid scintillation counting.

\section{Determination of Somatostatin Level}

For somatostatin determination, an aliquot of the homogenate was mixed with $4 \mathrm{~N}$ acetic acid ( $\mathrm{vol} / \mathrm{vol})$, centrifuged at $28,000 \mathrm{~g}$ at $4^{\circ} \mathrm{C}$ for $10 \mathrm{~min}$, and the supernatant was lyophilised. Somatostatin was measured by radio-immunoassay using the antibody M-6 [35] and iodinated N-Tyr or Tyr ${ }^{1}$ somatostatin as tracer [36].

Data Analyses

All data are given as means \pm SEM. For statistical analyses, the one-way ANOVA was applied. A Student $t$ test was used, where appropriate. Asterisks indicate a significant difference: $* \mathrm{p}<0.05$; ** $\mathrm{p}<0.01 ; * * * \mathrm{p}<0.001$ compared to control.

\section{Results}

\section{Behaviour}

Rats with KA-induced seizures, i.e. rating scale scores $3.36 \pm 0.15(\mathrm{n}=11)$ and $\operatorname{WDS} 68.64 \pm 17.8(\mathrm{n}=11)$, recovered within 2 weeks and then were seizure free; however, during the first, second and third month, myoclonic movements (focal convulsions) occurred (table 1). One month after KA injection, spontaneous seizures were observed. Up to the fifth month 1-2 and during the sixth month 3-5 spontaneous seizures per month and rat were observed. A progressive decline of focal convulsions and a progressive, but fluctuating enhancement of spontaneous seizures, in parallel with an increase in the time period after KA administration could be observed (table 1). Oneway ANOVA revealed a significant effect of time period after KA injection on focal convulsions and spontaneous seizure occurrences $(F=9.529, p=0.006 ; F=160.672$, $\mathrm{p}<0.0019$; table 1). Each of the KA-treated rats showed 5-9 WDS per month. Clinically, apparent seizure activities, manifesting as whisker twitching, tail extension, body twisting and body rearing with pedalling of the forepaws were observed. Spontaneous seizures lasted from 5 to $15 \mathrm{~s}$. Generally, the animals were aggressive on handling, particularly on touching their backs (very sensitive part pointing to pain induction). The control animals did not show WDS or any epileptic symptoms.

\section{Neurochemical Changes}

In control animals, the activities of ChAT and GAD and the somatostatin level in the frontal, cingulate, occipital, parietal and temporal cortices, the amygdala/piriform cortex, hippocampus, caudate nucleus and substantia nigra showed an uneven regional distribution in the brain (table 2). Data are in line with previously published studies $[4,16,37]$. 


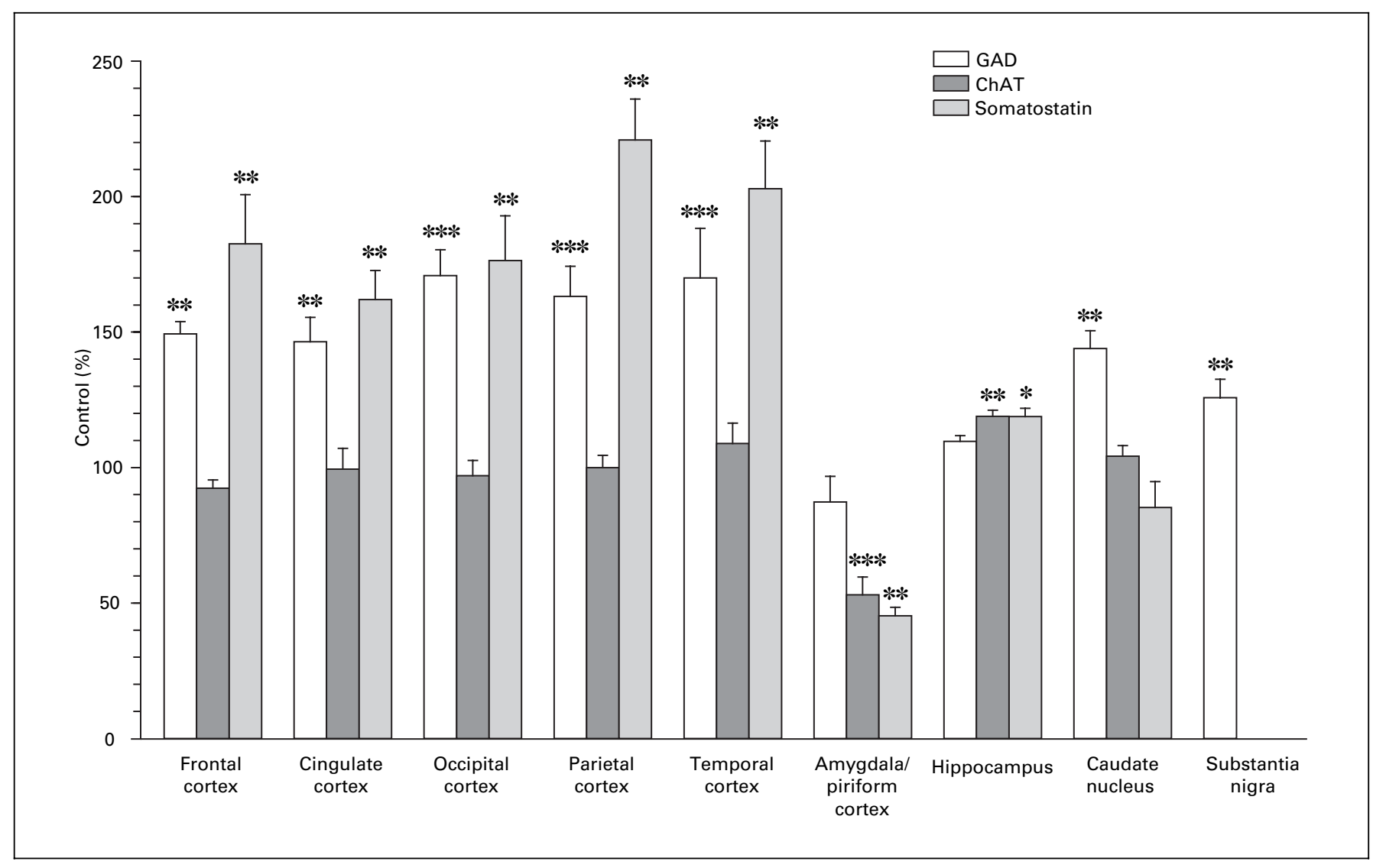

Fig. 1. Activities of GAD and ChAT and somatostatin levels in different rat brain regions in the chronic KA epilepsy model, 6 months after KA injection $(10 \mathrm{mg} / \mathrm{kg}$, s.c.). Data are expressed in percent of control and represent mean \pm SEM of 11 KA-treated animals. Significance of differences vs. the corresponding control group: ${ }^{*} \mathrm{p}<0.05 ;{ }^{* *} \mathrm{p}<0.01$; $* * * \mathrm{p}<0.001$ using the unpaired Student $t$ test.

Table 2. Control values of GAD and ChAT activities and somatostatin levels in different rat brain regions

\begin{tabular}{llcl}
\hline Region & $\begin{array}{l}\text { GAD } \\
\text { nmol/mg wet tissue } \\
\text { weight/h }\end{array}$ & $\begin{array}{l}\text { ChAT } \\
\text { nmol/mg wet tissue } \\
\text { weight/h }\end{array}$ & $\begin{array}{l}\text { Somatostatin } \\
\text { fmol/mg wet tissue } \\
\text { weight }\end{array}$ \\
\hline Frontal cortex & $19.23 \pm 0.56$ & $6.32 \pm 0.44$ & $64.6 \pm 3.4$ \\
Cingulate cortex & $17.65 \pm 0.67$ & $7.15 \pm 0.12$ & $99.3 \pm 9.4$ \\
Occipital cortex & $16.25 \pm 0.46$ & $5.34 \pm 0.19$ & $64.4 \pm 6.4$ \\
Parietal cortex & $18.34 \pm 0.33$ & $5.74 \pm 0.28$ & $49.7 \pm 3.8$ \\
Temporal cortex & $20.30 \pm 0.26$ & $5.86 \pm 0.08$ & $62.5 \pm 5.8$ \\
Amygdala/piriform cortex & $17.03 \pm 0.76$ & $13.91 \pm 0.40$ & $43.4 \pm 3.3$ \\
Hippocampus & $17.12 \pm 0.37$ & $7.10 \pm 0.17$ & $39.6 \pm 2.8$ \\
Caudate nucleus & $16.57 \pm 0.40$ & $26.46 \pm 0.99$ & $47.3 \pm 4.3$ \\
Substantia nigra & $66.01 \pm 4.82$ & $\mathrm{NE}$ & $\mathrm{NE}$ \\
\hline
\end{tabular}

Data represent means \pm SEM of 10 animals. $\mathrm{NE}=$ Not estimated. 
Table 3. Somatostatin:GAD ratio in different rat brain regions in the chronic KA epilepsy model 6 months after KA administration $(10 \mathrm{mg} / \mathrm{kg}$, s.c. $)$

\begin{tabular}{lc}
\hline Region & $\begin{array}{l}\text { Somatostatin:GAD } \\
\text { ratio, \% of control }\end{array}$ \\
\hline Frontal cortex & 122 \\
Cingulate cortex & 110 \\
Occipital cortex & 103 \\
Parietal cortex & 130 \\
Temporal cortex & 119 \\
Amygdala/piriform cortex & 51 \\
Hippocampus & 108 \\
Caudate nucleus & 59 \\
Substantia nigra & $\mathrm{NC}$ \\
\hline
\end{tabular}

Ratio was calculated between somatostatin and GAD levels in brain regions of KA-treated and control animals and data are expressed in percent of control. $\mathrm{NC}=$ Not calculated.

Six months after KA injection, the ChAT activity was reduced in the amygdala/piriform cortex (53\% of control; $\mathrm{p}<0.001)$, while it was moderately increased in the hippocampus ( $119 \%$ of control; $p<0.01$ ) (fig. 1$)$ and no alteration of ChAT activity was seen in cortical regions and caudate nucleus. Six months after KA injection, the GAD activity was increased in the occipital (171\% of control; $\mathrm{p}<0.001)$, temporal $(170 \%$ of control; $\mathrm{p}<0.001)$, parietal ( $163 \%$ of control; $\mathrm{p}<0.001)$, frontal $(149 \%$ of control; $\mathrm{p}<$ $0.01)$ and cingulate cortices $(146 \%$ of control; $\mathrm{p}<0.01)$ (fig. 1). An increase in GAD activity was also measured in the caudate nucleus $(144 \%$ of control; $\mathrm{p}<0.001)$ and the substantia nigra ( $126 \%$ of control; $p<0.01)$, whereas in the amygdala/piriform cortex, GAD activity was moderately lowered and in the hippocampus, it was comparable to the control animals. An increased somatostatin level was found in the parietal $(221 \%$ of control; $p<0.001)$, temporal $(203 \%$ of control; $p<0.001)$, frontal $(183 \%$ of control; $\mathrm{p}<0.001)$, occipital ( $176 \%$ of control; $\mathrm{p}<0.001$ ) and cingulate cortices $(162 \%$ of control; $p<0.001)$ and in the hippocampus $(119 \%$ of control; $p<0.05)$ (fig. 1$)$. The somatostatin level was considerably lowered in the amyg$\mathrm{dala} /$ piriform cortex $(45 \%$ of control; $\mathrm{p}<0.001)$ and moderately lowered in the caudate nucleus.

\section{Somatostatin:GAD Ratio}

Six months after KA injection, the somatostatin:GAD ratio was reduced in the amygdala/piriform cortex (51\% of control) and in the caudate nucleus (59\% of control) and moderately increased in the parietal $(130 \%$ of control), frontal (122\% of control) and temporal cortices (119\% of control) (table 3 ). In the cingulate and occipital cortices and in the hippocampus, the somatostatin:GAD ratio was comparable with that of the control animals.

\section{Correlation between Seizure Severity and ChAT Activity and Somatostatin Levels}

Significant differences in the ChAT activity and somatostatin level in the amygdala/piriform cortex have been found between KA-treated rats of rating 3 and rating 4, 6 months after KA injection (table 4). Rats with higher seizure activity in the 6th month of the study showed significantly decreased ChAT activity in the amygdala/piriform cortex. Similarly, the somatostatin levels in the amygdala/piriform cortex were also significantly decreased in rats with higher seizure activity. A negative relationship was found between changes of ChAT activity in amygdala/piriform cortex and the rating of KA-treated animals $(\mathrm{R}=-0.9372 ; \mathrm{p}<0.0001)$ or the number of spontaneous seizures observed during the last month (6th) after KA injection $(R=-0.73366 ; p=0.01017)$. Similarly, a negative relationship was also found between changes of somatostatin levels in the amygdala/piriform cortex and the rating of KA-treated animals $(\mathrm{R}=-0.8748$; $\mathrm{p}<$ 0.0001 ) or the number of spontaneous seizures observed during the last month (6th) after KA injection $(\mathrm{R}=$ $-0.82226 ; p=0.00189)$.

\section{Discussion}

Within 6 months after the initial KA-induced seizures, the rats developed repetitive seizures, the number of which was fluctuating. The neurochemical investigation has revealed increased GAD activity in all cortical brain regions as well as in the caudate nucleus and the substantia nigra. The ChAT activity was only increased in the hippocampus and significantly lowered in the amygdala/ piriform cortex, whereas the somatostatin levels were increased in all cortical regions and in the hippocampus, but reduced in the amygdala/piriform cortex. These neurochemical alterations in the brain 6 months after KA injection show a pattern that is different from the alteration observed after the initial KA-induced seizures [4, 5, 11-16]. Interestingly, the reduction of ChAT activity and of the somatostatin levels in the amygdala/piriform cortex 6 months after KA injection significantly correlates with the severity of the initial induced seizures and is in line with the high number of spontaneous seizures occurring 
Table 4. ChAT and somatostatin levels in the amygdala/piriform cortex in rats with different seizure activities 6 months after KA injection

\begin{tabular}{|c|c|c|c|}
\hline \multicolumn{2}{|l|}{ Behaviour } & \multirow{2}{*}{$\begin{array}{l}\mathrm{ChAT} \\
\mathrm{nmol} / \mathrm{mg} \text { wet tissue } \\
\text { weight } / \mathrm{h}\end{array}$} & \multirow{2}{*}{$\begin{array}{l}\text { Somatostatin } \\
\text { fmol/mg wet tissue weight }\end{array}$} \\
\hline $\begin{array}{l}\text { rating of rats within } 2 \mathrm{~h} \\
\text { after KA injection }\end{array}$ & $\begin{array}{l}\text { spontaneous seizures } \\
\text { during the last month } \\
\text { (6th) after KA injection }\end{array}$ & & \\
\hline $3.00 \pm 0.00(7)$ & $3.00 \pm 0.00(7)$ & $\begin{array}{l}8.66 \pm 0.12(7)^{b} \\
62 \% 1\end{array}$ & $\begin{array}{l}21.53 \pm 0.52(7)^{b} \\
52 \% 0^{1}\end{array}$ \\
\hline $4.00 \pm 0.00(4)^{\mathrm{a}}$ & $4.25 \pm 0.25(4)^{\mathrm{a}}$ & $\begin{array}{l}5.47 \pm 0.49(4)^{a, b} \\
39 \%{ }^{1}\end{array}$ & $\begin{array}{l}17.65 \pm 0.13(4)^{a, b} \\
41 \% 1\end{array}$ \\
\hline
\end{tabular}

The data present means \pm SEM. The number of animals is given in parenthesis. For behavioural rating, see Materials and Methods. The control value of ChAT activity and somatostatin level in the amygdala/piriform cortex was $13.91 \pm 0.40 \mathrm{nmol} / \mathrm{mg}$ wet tissue weight $/ \mathrm{h}$ and $43.4 \pm 3.3 \mathrm{fmol} / \mathrm{mg}$ wet tissue weight, respectively. Significance using Student's t test: ${ }^{\mathrm{a}} \mathrm{p}<0.001$ vs. KA-treated rats with rating $3.00 \pm 0.00 ;{ }^{\mathrm{b}} \mathrm{p}<0.001$ vs. control animals.

1 Percent of control.

during the 6th month of the experiment. This marked reduction of ChAT activity and somatostatin level in the amygdala/piriform cortex may be due to a loss of neurons containing acetylcholine and somatostatin; however, it could also be attributable to the extensive tissue damage [4].

On the other hand, an increase in ChAT activity in the hippocampus 6 months after KA injection could at least partially be attributable to the sprouting of cholinergic fibres, e.g. in the dentate gyrus. Indeed, our finding of increased ChAT activity in the hippocampus of the KA chronic temporal epilepsy corresponds to the findings of increased expression of both cholinergic fibre and mossy fibre sprouting into the inner molecular layer of the dentate gyrus following KA-induced seizures [38]. It is interesting to note that the increased ChAT activity in the hippocampus was accompanied by an increase in the somatostatin level. The reorganisation of different fibre systems including those derived from GABAergic and cholinergic neurons is already recognised [17, 38]. It is therefore very likely that the type and extent of sprouting ultimately modify the hippocampal excitability. Somatostatin has already been suggested as a possible excitatory transmitter in the hippocampus [27]. According to the study performed by Higuchi et al. [28], the administration of cysteamine, a compound which reduces the central somatostatin content, had a profound anticonvulsive effect in pentylenetetrazol-kindled rats. There are also other significant findings, which indicate that somatostatin may reduce seizure activity and inhibit neurons. Vezzani et al. [29] reported that the infusion of a peptidase-resistant somatostatin agonist during quinolinate- and KAinduced convulsions resulted in a reduction of seizure activity. However, the mechanism that initiates and mediates the cholinergic and the hypothetical somatostatinergic axonal sprouting in the hippocampus following repetitive seizures has not been found out so far.

It is remarkable that the magnitude of the increase in GAD activity in all cortical brain regions is very similar to that of the increase in somatostatin 6 months after KA injection. Sustained increase in the inhibitory neurotransmitter GABA by increased activities of the synthesizing enzyme GAD can represent an endogenous anticonvulsive mechanism. The effectiveness of the GABAergic and somatostatinergic systems in modulating the seizure activities has been demonstrated by pharmacological approaches in the acute KA-induced epilepsy [29, 37, 39, 40]. However, the pathophysiological mechanism underlying increased synthesis of neuropeptide contained within GABAergic neurons in cortical brain regions 6 months after KA has not been clarified yet. In the chronic KA-induced epilepsy model, hyperactivity of GABAergic neurons in the nigrostriatal pathway might also suppress the occurrence of spontaneous seizures. There are significant data showing that the inhibition of neuronal activity in the substantia nigra by GABAergic agonists leads to the suppression of tonic epileptic events of generalised convulsions [41, 42]. Turski et al. [43] demonstrated that a selective activation of excitatory amino acid receptors in the substantia nigra reticulata considerably lowers the 
threshold for induced seizures. On the other hand, it is reasonable to question to what extent an increased activity of the GABAergic system may protect the brain from excitotoxic processes, since in cortical cultures a cell death acceleration after adding GABA has been found [44]. The wide variation of disturbances of the neuronal balance between excitatory and inhibitory neurotransmitters and/ or between neuro-active compounds may be the main cause of the complex pathological process in the epileptic brain [6]. In our study, the somatostatin:GAD ratio was reduced in the amygdala/piriform cortex and in the caudate nucleus and was moderately increased in other brain areas. Although the mechanisms that initiate and mediate axonal sprouting following seizures are still unknown, it has been suggested that endogenous nerve growth factors play a functional role in mediating permanent structural changes and permanent biochemical alterations following seizures [28]. The authors have demonstrated that intraventricular infusion of a nerve growth factor-specific anti- body, which blocks nerve growth factor biological activity in vitro, attenuated the cholinergic axonal sprouting of basal forebrain cholinergic neurons following seizureinduced injury in vivo. In the future, experiments with GABA-mimetic drugs or drugs characterised by N-methyl- $D$-aspartate receptor-mediated release of GABA [45] or even reagents that influence the sprouting of cholinergic and GABAergic neurons will be of particular significance in order to prove their anti-epileptic properties and capability to modulate the progression of neural degeneration in the chronic KA model for epilepsy and may give new insight into the treatment of chronic epilepsy.

\section{Acknowledgements}

This work was supported in part by the Austrian Science Research Project FWF P15371 and was performed in part at the Institute of Biochemical Pharmacology, University Vienna.

\section{References}

1 Pisa M, Sanberg PR, Corcoran ME, Fibiger $\mathrm{HC}$ : Spontaneously recurrent seizures after intracerebral injection of kainic acid in rat: A possible model of human temporal lobe epilepsy. Brain Res 1980;200:481-487.

$>2$ Olney JW, Rhee V, Ho OL: Kainic acid: A powerful neurotoxic analogue of glutamate. Brain Res 1974;77:507-512.

-3 Nadler JV: Kainic acid as a tool for the study of temporal lobe epilepsy. Life Sci 1981;29:20312042.

4 Sperk G, Lassmann H, Baran H, Kish SJ, Seitelberger F, Hornykiewicz O: Kainic acid induced seizures: Neurochemical and histopathological changes. Neuroscience 1983;10:13011315.

$>5$ Ben-Ari Y: Limbic seizure and brain damage produced by kainic acid: Mechanisms and relevance to human temporal epilepsy. Neuroscience 1985;14:375-403.

6 Meldrum B: Excitotoxicity and epileptic brain damage. Epilepsy Res 1991;10:55-61.

7 Löscher W: Basic aspects of epilepsy. Curr Opin Neurol Neurosurg 1993;6:223-232.

$>8$ Watkins JC, Krogsgaard-Larsen P, Honore T: Structure-activity relationships in the development of excitatory amino acid receptor agonist and competitive antagonist. Trends Pharmacol Sci 1990;11:25-33.

9 Schwarcz R, Zaczek R, Coyle JT: Microinjection of kainic acid into the rat hippocampus. Eur J Pharmacol 1978;50:209-220.
10 Schwob JE, Fuller T, Price JL, Olney JW: Widespread patterns of neuronal damage following systemic or intracerebral injections of kainic acid: A histological study. Neuroscience 1980;5:991-1014.

11 Heggli DE, Aamodt A, Malthe-Sörenssen D: Kainic acid neurotoxicity; effect of systemic injection on neurotransmitter markers in different brain regions. Brain Res 1981;230:253262.

12 Heggli DE, Malthe-Sörenssen D: Systemic injection of kainic acid: Effect on neurotransmitter markers in piriform cortex, amygdaloid complex and hippocampus and protection by cortical lesioning and anti-convulsants. Neuroscience 1982; 7:1257-1264.

13 Lothman EW, Collins RC: Kainic acid induced limbic seizures: Metabolic, behavioural, electroencephalographic and neuropathological correlates. Brain Res 1981;218:299-318.

$\checkmark 14$ Baran H, Heldt R, Hertting G: Increased prostaglandin formation in rat brain following systemic application of kainic acid. Brain Res 1987;404:107-112.

15 Baran H: Changes of dopaminergic neurotransmission after kainic acid-induced behavioural alteration. Soc Neurosci 1994;20:593.7.

16 Sperk G, Wieser R, Widmann R, Singer EA: Kainic acid induced seizures: Changes in somatostatin, substance $P$ and neurotensin. Neuroscience 1986;17:1117-1126.

17 Davenport CJ, Brown WJ, Babb TL: Sprouting of GABAergic and mossy fiber axons in dentate gyrus following intrahippocampal kainate in the rat. Exp Neurol 1990;109:180-190.
18 Feldblum A, Ackermann RF, Tobin AJ: Longterm increase of glutamate decarboxylase mRNA in a rat model of temporal lobe epilepsy. Neuron 1990;5:361-371.

19 Marksteiner J, Sperk G: Concomitant increase of somatostatin, neuropeptide Y, and glutamate decarboxylase in the frontal cortex of rats with decreased seizure threshold. Neuroscience 1988;26:379-385.

20 McGeer PL, McGeer EG, Wada JA: Glutamic acid decarboxylase in Parkinson's disease and epilepsy. Neurology 1971;21:1000-1007.

21 Lloyd KG, Bossi L, Morselli PL, Munari C, Rougier M, Loiseau H: Alterations of GABAmediated synaptic transmission in human epilepsy. Adv Neurol 1986;44:1033-1044.

22 Nadi NS, Porter RJ, Wyler AR: Choline acetyltransferase, glutamate decarboxylase and tyrosine hydroxylase in the human epileptic focus. Neurology 1987;37(suppl 1):106.

23 Babb TL, Pretorius JK, Kupfer WR, Crandall PH: Glutamate decarboxylase-immunoreactive neurons are preserved in human epileptic hippocampus. J Neurosci 1989;9:2562-2574.

24 Cavalheiro EA, Riche DA, DeGal LaSalle G: Long-term effects of intrahippocampal kainic acid injection in rats: A method for inducing spontaneous recurrent seizures. Electroencephalogr Clin Neurophysiol 1982;53:581-589.

25 Tanaka T, Kaijima M, Yonemasu Y, Cepeda $\mathrm{C}$ : Spontaneous secondarily generalized seizures induced by a single microinjection of kainic acid into unilateral amygdala in cats. Electroencephalogr Clin Neurophysiol 1985; 61:422-429. 
26 Hendry SHC, Jones EG, DeFelipe J, Schmechel D, Brandon C, Emson PC: Neuropeptidecontaining neurons of the cerebral cortex are also GABAergic. Proc Natl Acad Sci USA 1984;81:6526-6530.

27 Dood J, Kelly JS: Is somatostatin an excitatory transmitter in the hippocampus? Nature 1978; 273:674-675.

28 Higuchi T, Sikand GS, Kato N, Wada JA Friesen HG: Profound suppression of kindled seizures by cysteamine: Possible role of somatostatin to kindled seizures. Brain Res 1983; 288:359-362.

29 Vezzani A, Sefarini R, Stasi MA, Vigabno G, Rizzi M, Samanin R: A peptidase-resistant cyclic octapeptide analogue of somatostatin (SMS 201-995) modulates seizures induced by quinolinic and kainic acids differentially in the rat hippocampus. Neuropharmacology 1991;30: 345-352.

30 Baran H, Lassmann H, Hornykiewicz O: Behavior and neurochemical changes 6 months after kainic acid. Soc Neurosci 1988;14:593.7.

31 König JFR, Klippel RA: The Rat Brain, a Stereotactic Atlas of the Forebrain and Lower Parts of the Brain Stem. Huntington, RE Krieger, 1970.

32 Skinner JE: Neuroscience: A Laboratory Manual. Philadelphia, Waunders Company 1971, pp 198-199.
33 Roberts E, Simonsen DG: Some properties of $L$-glutamic decarboxylase in mouse brain. Biochem Pharmacol 1963;12:113-134.

34 Fonnum F: Radiochemical microassays for the determination of choline acetyltransferase and acetylcholinesterase activities. Biochem J 1969;115:465-472.

-35 Arnold MA, Fernstrom JD: Administration of anti-somatostatin serum to rats reverses the inhibition of pulsatile growth hormone secretion produced by injection of metergoline but not yohimbine. Neuroendocrinology 1980;31: 194-199.

6 Gamse R, Leeman SE, Holzer P, Lembeck F: Differential effects of capsaicin on the content of somatostatin, substance $\mathrm{P}$ and neurotensin in the nervous system of the rat. Naunyn Schmiedebergs Arch Pharmacol 1981;317: 140-148.

>37 Baran H, Sperk G, Hörtnagl H, Sapetschnig G, Hornykiewicz O: $\alpha_{2}$-Adrenoceptors modulate kainic acid-induced limbic seizures. Eur J Pharmacol 1985;113:263-269.

38 Holzman DM, Lowenstein DH: Selective inhibition of axon outgrowth by antibodies to NGF in a model of temporal lobe epilepsy. J Neurosci 1995;15:7062-7070.

39 Baran H, Löscher W, Mevissen M: The glycine/ NMDA receptor partial agonist $D$-cycloserine blocks kainate-induced seizures in rats. Comparison with MK-801 and diazepam. Brain Res 1994;652:195-200.
40 Ben-Ari Y, Tremblay E, Ottersen OP, Naquet R: Evidence suggesting secondary epileptogenic lesions after kainic acid: Pre-treatment with diazepam reduces distant but not local damage. Brain Res 1979;165:362-365.

41 Iadarola MJ, Gale K: Substantia nigra: Site of anticonvulsant activity mediated by gammaaminobutyric acid. Science 1982;218:12371240.

42 Depanulis A, Vergnes M, Marescaux C: Endogenous control of epilepsy: The nigral inhibitory system. Prog Neurobiol 1994;42:33-52.

43 Turski L, Ikonomidou C, Turski WA, Bortolotto ZA, Cavalheiro EA: Cholinergic mechanisms and epileptogenesis. The seizures induced by pilocarpine: A novel experimental model of intractable epilepsy. Synapse 1989;3: 154-171.

44 Erdo SL, Michler A, Wolff JR: GABA accelerates excitotoxic cell death in cortical cultures: Protection by blockers of GABA-gated $\mathrm{Cl}^{-}$ channels. Brain Res 1991;542:254-258.

45 Baran H, Gramer M, Löscher W: Alterations in plasma and brain amino acids after administration of the glycine/NMDA receptor partial agonist, $D$-cycloserine, to mice and rats. Eur $\mathrm{J}$ Pharmacol 1995;273:197-201. 